\title{
Analyzing the Effects of Reinforcement Learning to Develop Humanoid Robots
}

\author{
Naaima Suroor, Jamia Hamdard, New Delhi, India \\ Imran Hussain, Jamia Hamdard, New Delhi, India \\ Aqeel Khalique, Jamia Hamdard, New Delhi, India \\ Tabrej Ahamad Khan, Jamia Hamdard, New Delhi, India
}

\begin{abstract}
Reinforcement learning is a flourishing machine learning concept that has greatly influenced how robots are designed and taught to solve problems without human intervention. Robotics is not an alien discipline anymore, and we have several great innovations in this field that promise to impact lives for the better. However, humanoid robots are still a baffling concept for scientists, although we have managed to develop a few great inventions which look, talk, work, and behave very similarly to humans. But, can these machines actually exhibit the cognitive abilities of judgment, problemsolving, and perception as well as humans? In this article, the authors analyzed the probable impact and aspects of robots and their potential to behave like humans in every possible way through reinforcement learning techniques. The paper also discusses the gap between 'natural' and 'artificial' knowledge.
\end{abstract}

\section{KEYWORDS}

Artificial Intelligence, Cognitive Thinking, Machine Learning, Reinforcement Learning, Robots 


\section{INTRODUCTION}

There was once a time in history when people could not have imagined sitting idly on their couch and getting information about almost anything that came across their minds with a few simple touches on a little device in their hand. Even the idea of a machine that could do all of our computational work in a matter of seconds was unfathomable.

But we are now living in a century where these technological dreams can be brought to life. Nothing seems impossible now. From health care to education, science, agriculture, and almost every possible discipline out there, there are innovations and newer technologies coming up each day to make people's lives easier and more efficient. The study of robotics is something very complex, but at the same time, equally intriguing and fruitful. The entire world could change exponentially if a day comes when people have successfully developed robots that are not just meant for jobs like cleaning and cooking, but ones that look, learn, and behave just like humans. Imagine the amount of time people would save if these machines could do all of the tedious, manual work that people typically do. Honestly, it would be a little frightening too, since most people have watched or heard of at least one movie where machines have turned against humans and taken over the world. It would also be unfair to acknowledge the various attempts that have been made over the years at creating humanoid robots that are similar to humans in some of their attributes. Modern robots are mostly capable of handling repetitive jobs that do not really require intelligence or decision making. Also, the majority of robots are limited to labs and manufacturing units, and they are not really competitive enough to be able to work in the real world. Working in a natural environment with several factors that affect a person's decisions and requires thinking spontaneously; these characteristics are difficult for robots because they are working based on a set of algorithms and can easily get confused in real-world situations (Rivlin, 2019).

There have been some fascinating discoveries in this field, but the one common feature that lacked in all of them was that of judgment or what has been famously coined as "theory of mind" (Scassellati, 2002). Another term that could explain this is self-awareness. The machine's source of information recognition and sensing relies on human input (Krening \& Feigh, 2018), and they require datasets for reference. For example, for a robot to identify a ball, humans provide it with several pictures of balls so that it can relate those images to identify one when it sees it. In the end, it comes down to a series of $0 \mathrm{~s}$ and $1 \mathrm{~s}$, which is the only thing robots are programmed to comprehend (Lauckner \& Lintner, n.d.). How do people teach robots to do complex actions? Robots are used to 'supervised learning', which means that they are aware of the output they should expect during any given task for specific input. This is where reinforcement learning comes in. Reinforcement learning is associated with deep learning that teaches systems to solve problems using trial and error methods. It is used when complex decision making is required and is therefore iterative in nature due to the multiple training algorithms it uses to come to a solution. This is because the environment it is dealing with is dynamic or unstructured in nature. The 
environment that the robot is adapted to work in is a structured environment where everything is ordered and in place (Govers, 2018). Thus, this technique is more time consuming because, for every given task, all possible scenarios are checked to obtain a result. But, for the same reason, it has a higher accuracy rate. Therefore, it is being heavily researched and implemented in robotics. Next, the authors provide a deeper understanding of this discipline.

\section{USE OF ARTIFICIAL INTELLIGENCE (AI) IN DEVELOPMENT OF INTELLIGENT MACHINES}

In layman's terms, artificial intelligence (AI) explains how machines can be taught to think for themselves. AI was originated in 1965, but the field has recently gained the curiosity of scientists. Research and inventions regarding AI are helping to determine the scope of AI development. AI is used in healthcare, manufacturing, and banking. It is broadly translated into two categories: strong AI and weak AI. Strong AI is when machines have the ability to carry out tasks on their own whereas weak AI requires human intervention (Rouse et al., 2018). AI assists humans in many ways. Technologies like automation, neural networks, deep learning (used interchangeably with machine learning but is also applicable for larger datasets), robotics, machine learning, and Natural Language Processing (NLP) are few of the major applications of AI that are in demand. Cognitive computing is the subfield that would have a path-breaking impact in making human-like interactions possible. Unfortunately, there has not been much development in the field, and it is still a work in progress.

There are also several types of AI that can help understand AI. Modern machines can be broadly termed as reactive machines, or as machines with limited memory, which are self-aware and have a theory of mind (Azarian, 2016). The latter two are something that does not currently exist. Modern machines cannot learn from their mistakes like humans; they need a massive amount of complex data to be able to recognize objects and make decisions by formulating predictive models (Data Science Graduate Programs, n.d.). Machine learning is a part of AI where robotics and data science meet (Domingos, 2012).

\section{USING MACHINE LEARNING TO CREATE FUTURISTIC ROBOTS}

The meaning of machine learning (ML) is pretty self-explanatory; it refers to machines that teach themselves and have the ability to learn on their own without the help of humans or tasks being explicitly programmed. They learn to do their allotted work by generalizing from examples (Raj \& Seamans, 2019). ML has been classified as a narrow AI, meaning that it makes predictions for the future based on pre-existing data using sophisticated and complex algorithms, as displayed in Figure 1 (Jones et al., 2018).

Machine learning is the amalgamation of robotics and data science. Data science is a mix of various disciplines blended together to obtain meaningful data from humongous amounts of raw data such as statistics, algorithms, ML, data analysis, and other related disciplines. Robotics is the study of making machines (robots) that can 
Figure 1. Understanding the relationship between $\mathrm{ML}$ and $\mathrm{Al}$ [image source: https://data-flair.training/blogs/machinelearning-tutorial/]

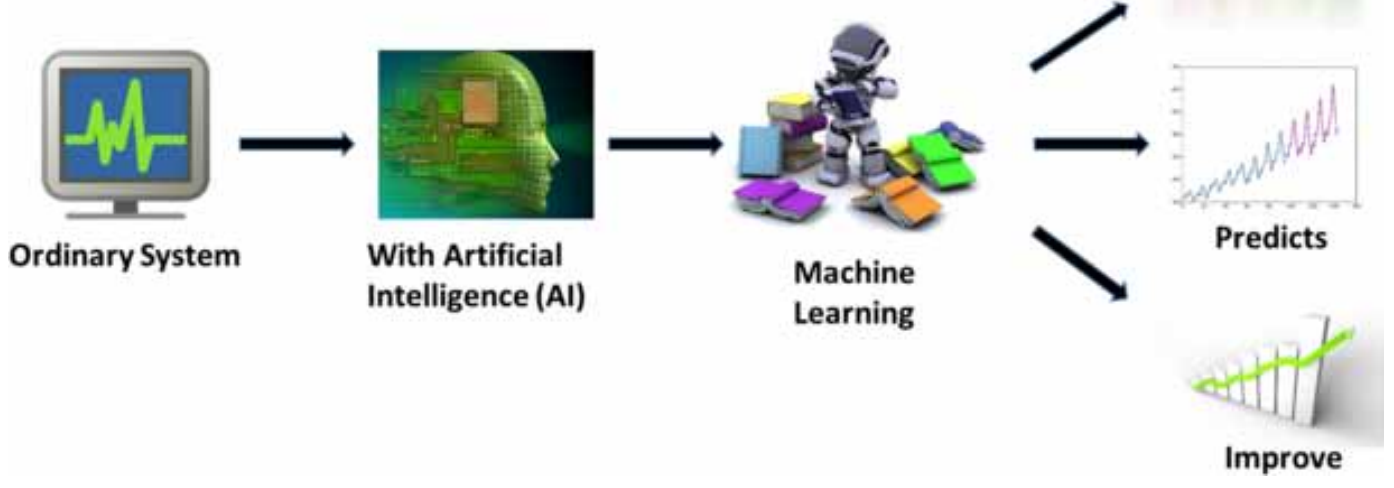

assist with tasks that were previously done manually by humans. Together, these two sciences transform into ML. ML is thus a highly useful field that can make machines manipulate and move objects, as well as learn to perceive, reason, and plan for better problem-solving. ML has proved to benefit humans in a lot of ways. With respect to robots, ML has made the future easier by being able to do extensive manual labor that previously had to be done by humans. This makes work more secure since it is less likely for a machine to make a mistake. Having a robot that cooks exotic cuisines and cleans up dishes is something everyone would want (Kidd \& Breazeal, n.d.). But these are just monotonous tasks where no decision making is involved.

To think of futuristic robots, the need for them to possess cognitive abilities to be able to sense a problem and take appropriate actions to deal with it needs to be addressed. A mechanized machine would not be able to sense and make out the graveness of an issue or take the most efficient action. This could prove to be harmful in some respects. For instance, what if the machine decides that unemployed people in a region or a country are a liability to its growth and economy (Schuman, 2015) and decides the best solution is to throw them out or even worse, eliminate them. This is a situation that is not likely to happen, but if the control of the machines is left completely on themselves, then it would not be a surprise that they would do something like this. Thus, cautious steps need to be taken in this field.

\section{LEARNING STYLES IN ML AND DEEPER INSIGHT INTO REINFORCEMENT LEARNING}

Machine learning provides three important learning algorithms: supervised learning, unsupervised learning, and reinforcement learning.

In supervised learning, the dataset is labeled. It is the most commonly used algorithm out of the three and exhibits precise mapping between the input and the output data. Some examples of supervised learning are linear regression and artificial 
neural networks. An important application of this learning has been facial recognition, which is now commonly used to unlock smartphones (Jones et al., 2018).

Unsupervised learning is a type of learning where the datasets are not labeled explicitly. Rather, implicit patterns are found in the data and the machine learns from it (Bible \& Dutta, 2013). Important examples of unsupervised learning are clustering and autoencoders. A well-known application of this is clustering used in businesses.

Reinforcement learning involves no training dataset. The machine reaches its goals by interacting with a dynamic environment. Unlike the other two where the robot or machine knew what output to expect, here the software must make observations in various situations and then take the most appropriate action for it in a specific environment. This maximizes the returns it has. In the real world, there are situations where sequential decisions need to be taken instead of just one.

Reinforcement learning along with deep learning and imitation learning has been studied to make robots act according to the ever-changing nature of the environment they are in. It has proved to be successful as well but only in controlled environments. To make it work in the real world, they need to develop common sense and more complex decision-making skills. Researchers are moving in the right direction with their experiments. Scientists are working on projects like the 'Blue Brain' which has been working to create a perfect working replica of the human brain (Sundaresan, 2016). If they are somehow victorious, then it would be the end of one of the biggest mysteries in the world. It has perplexed people for years, and if the human brain gets decoded, then it opens so many doors for new discoveries.

\section{PROBING THE POSITIVE OUTCOMES OF HAVING SELF-LEARNING ROBOTS}

If robots can start making decisions themselves, they could aid humans in important discussions about the future such as climate change, fighting crimes, and the falling economy. This is because an individual has a certain viewpoint that can get affected or remain very rigid due to several social and psychological factors. But for a machine, this does not prove to be a hurdle. They will make decisions that are unbiased and the most optimal for any given situation.

Tasks that are currently done manually and may be hazardous or backfire if any minor mistake is made (e.g., driving, surgeries, construction, etc.) would become perfectly efficient and error-free with robots. There would be no loss of life or property if machines were to do these instead of people. While self-driving cars and robots that assist in surgeries do exist, these tasks, for now, are heavily supervised. Only when control is given to robots that can think for themselves would humans actually get rest.

War is another area that robots could help with. War causes a huge loss of life and property, and many soldiers die while protecting their respective nations. If robots begin to be used in warfare, then no more innocent lives would be lost.

Robots could assist with education. Not only to the students who are perfectly healthy and physically available but also to the ones who might not be present in 
classes due to some disability or other constraints. Robots could also benefit autistic students who might have trouble communicating with people. Thus, they can act as great companions or friends.

Additionally, places that are hard to reach during natural calamities by humans would not be an issue anymore as robots could help provide relief to the needy and the wounded. They could also help in cleaning hazardous places, such as sewers, which take so many lives.

If robots were to become more like humans, many problems and difficulties faced today could come to an end. In addition to the previously discussed mundane jobs that robots of today (Figures 2 and 3) are already doing, there are limitless other areas that can be probed. There are endless possibilities as to what humans would be able to achieve with machines that think like them.

\section{ISSUES OR CHALLENGES WITH IMPLEMENTATION OF REINFORCEMENT LEARNING IN ROBOTS}

There are several loopholes to this study that cannot be ignored. Even though the idea of completely mechanizing human life seems glorious, it is also important to consider what would happen to the people who would lose their jobs if robots replaced them. For example, drivers livelihood, construction laborers, cooks, house helpers, and so many others would be left unemployed. It is difficult to imagine how new jobs would be created for them when they are already trained for their respective ones.

Imagining that the robots can be just like humans is one thing, but at the same time, reality seems a bit different. For instance, smart machines exist, but can they really possess the self-awareness, conscience, judgment, and problem solving of humans? Predicting other's intentions is not something robots can grasp. Algorithms can help them solve a task in millions of attempts. However, if they come across a situation that

Figure 2. Applications of the humanoid robot [image source: http://www.imedpub.com/articles-images/applied-scienceresearch-review-Humanoid-Robot-5-4-17-g001.png]

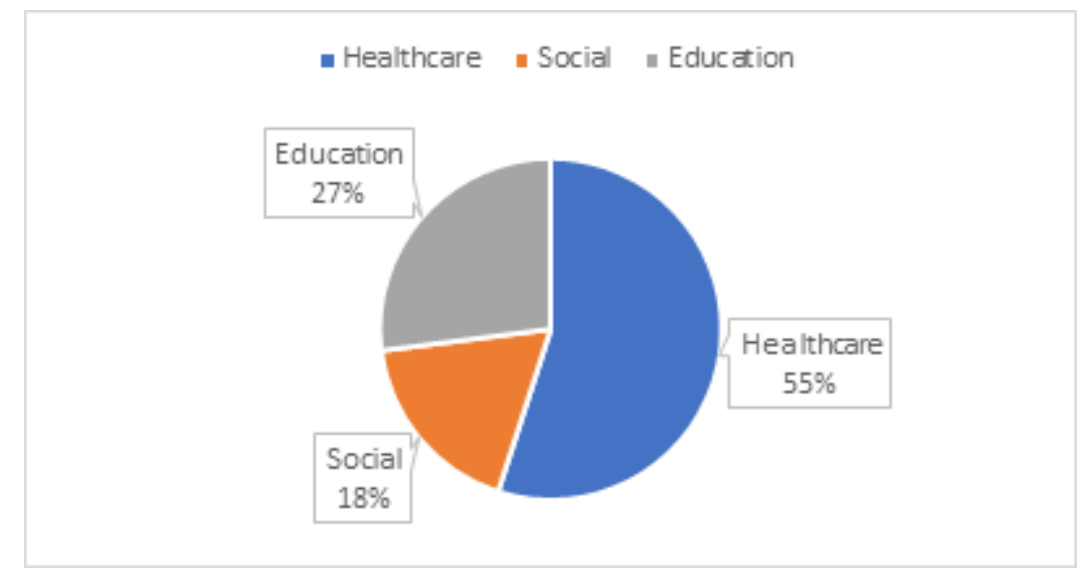


Figure 3. Different tasks humanoid robots can be used for [image source: https://www.toyota-global.com/innovation/ partner_robot/robot/images/mainimg01.jpg]

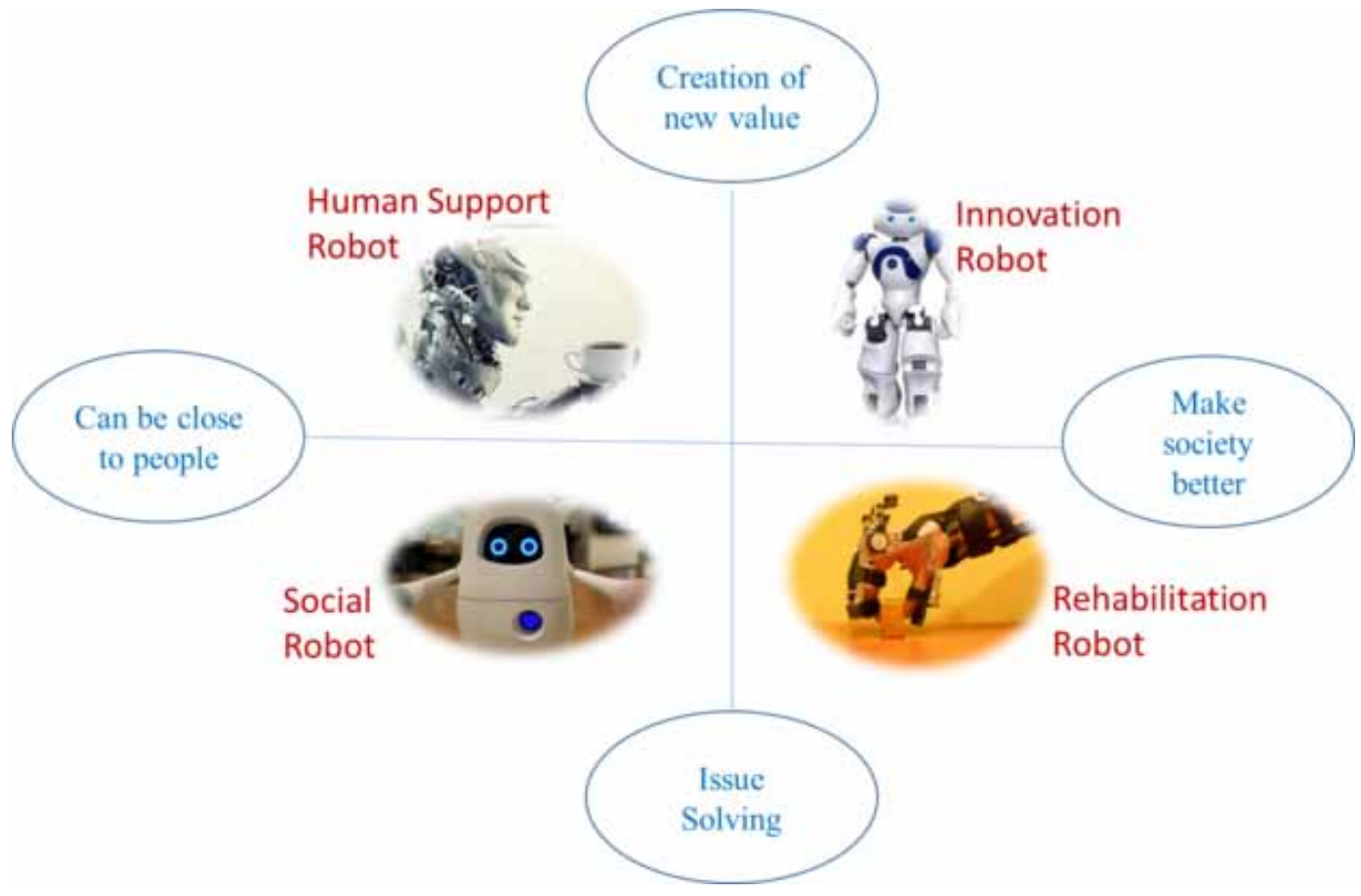

is completely alien to their system, then all that they have learned becomes useless. Lying, manipulation, and greed are not something they would be able to deal with.

The idea of driverless automobiles seems great, but in reality, it is ultimately a machine that is doing a job. Although the success rate of machines is much higher, if one fails, the results are much more catastrophic than human errors and can lead to the loss of lives (Sunderhauf et al., 2018).

Machines based on RL can make calculated decisions based on trial and error. Even for simple problems, they would run all possible outcomes of it. Thus, any task they do is error-free but very time-consuming. The more time that is taken to complete jobs, the more money that is used. Updating and maintenance would be costly too.

In surgeries, complications arise that are completely new and baffle doctors. In such cases, machines would not know what to do as the problem would not be something that they have faced before. By the time they do come up with a solution, it would prove to be fatal for the patient.

Cyber terrorism is a grave evil that the world is now facing. Robots are ultimately machines and if they are getting smarter, hackers and other wrongdoers also get smarter. In this sense, robots could be used against individuals or society for theft, terrorism, and other fraudulent activities. For example, hackers could hack machines used for providing security in homes and organizations, which could provide access to the building. Hackers could also meddle with the functions of a driverless automobile to cause accidents. 


\section{RELATED WORK AND IMPORTANT CONTRIBUTIONS TO THE FIELD}

While researchers are still trying to figure out if these humanoid robots will prove to be a boon or bane for mankind, the various attempts that are already being made throughout the world need to be acknowledged. Some of the important innovations in this field are discussed below.

In 2000, Honda created a robot named ASIMO, which was a two-legged robot with five finger-arms. It was a breakthrough invention and is currently preserved in a lab in Tokyo, Japan. ASIMO can recognize people, sounds, and its environment; it can even understand different languages. It can also respond to a handshake if initiated. Thus, it was a great new invention at its time (Hirose \& Ogawa, 2006).

ATLAS is another invention in the field of industrial robots and was created in 2013 by Boston Dynamics. It is a bipedal robot and can exhibit various loco-motor skills like running and backflips extremely well. It can also assist in construction (Kuindersma et al., 2016). Keeping its skills in mind, it could prove to be of great help during warfare.

Kengoro, created by the University of Tokyo can mimic human movements and even appears to sweat. Compared to humans, it has roughly three times the amount of freedom in its movements.

Sophia has been a very popular face among humanoids and was developed by Dr. Hanson. She can converse with humans, learn from their behavior, and experience and exhibit various facial gestures. It has been constantly improving as well (Retto, 2017).

Furthermore, NAO works with children that are autistic and runs sessions in caretaking homes. It was created in 2008 (Esteban et al., 2017).

Numerous others also exist, such as ERICA and Valkyrie along with notable non-humanoid bots such as PARO and BUDDY that assist humans and are examples of super-intelligent machines. The list goes on, but they still are not the robots that people envision, but they are still a step in the right direction.

\section{CONCLUSION}

In the end, there are still assumptions about the future of humanoid robots because they are still a work in progress. It would be a huge feat for scientists and the impact it has depends on the perception of the people. A boon for an individual may be considered a bane by someone else. Although there are several safety and time constraints, robots can help formulate a better future for mankind as each of these individual machines is the result of multiple genius brains. The only hurdle left is the time required for artificial intelligence to replicate natural intelligence as precisely as possible because scientists are still doubtful of the success of turning this dream into complete reality. Intensive research has been going on and has had ground-breaking discoveries that were unimaginable before. So, this gap can be diminished in the coming years.

People cannot look away from the fact that robots, whether humanoid or not, will have a role and be part of the future ahead. Even if exact replicas are not achieved, 
super-intelligent robots will compete with humans in several areas. Thus, humans and robots will need to develop a means for a healthy coexistence. Sooner or later, there is a high chance that with deep learning and imitation learning working alongside reinforcement learning, robots will not just imitate people, but they will actually learn to sustain themselves without a human teacher. 


\section{REFERENCES}

Azarian, B. (2016). To make robots more human-like, we need to teach them how to be mind readers. Quartz. Retrieved from https://qz.com/817476/to-make-robots-morehuman-like-we-need-to-teach-them-how-to-be-mind-readers/

Bible, J., \& Dutta, S. (2013). Informatics for materials science and engineering. Science Direct. Retrieved from https://www.sciencedirect.com/topics/chemical-engineering/ unsupervised-learning

Data Science Graduate Programs. (n.d.). Preparing for a data science career in AI and robotics R\&D with a master's degree. Retrieved from https://www. datasciencegraduateprograms.com/ai-and-robotics-rd/

Domingos, P. (2012). A few things to know about machine learning. Comm. of the ACM, 55(10).

Esteban, P. G., Baxter, P., Belpaeme, T., Billing, E., Cai, H., Cao, H. L., \& Fang, Y. et al. (2017). How to build a supervised autonomous system for robot-enhanced therapy for children with autism spectrum disorder. Paladyn : Journal of Behavioral Robotics, 8(1), 18-38. doi:10.1515/pjbr-2017-0002

Govers, F. X. (2018). Artificial intelligence for robotics: Build intelligent robots that perform human tasks using AI techniques. Birmingham, UK: Packt Publishing Ltd.

Hirose, M., \& Ogawa, K. (2007). Honda humanoid robots development. Philosophical Transactions of the Royal Society A: Mathematical, Physical and Engineering Sciences, 365(1850), 11-19.

Jones, L. D., Golan, D., Hanna, S. A., \& Ramachandran, M. (2018). Artificial intelligence, machine learning and the evolution of healthcare: A bright future or cause for concern? Bone \& Joint Research, 7(3), 223-225. doi:10.1302/2046-3758.73. BJR-2017-0147.R1 PMID:29922439

Kidd, C. D., \& Breazeal, C. (n.d.). Robots at home: Understanding long-term humanrobot interaction. Retrieved from http://srl.informatik.uni-freiburg.de/teachingdir/ ws 12/10-kiddIROS08.pdf

Krening, S., Karen, M., \& Feigh, K. M. (2018). Interaction algorithm effect on human experience with reinforcement learning. ACM Trans. Hum.-. Robot Interact, 7(2). doi:10.1145/3277904

Kuindersma, S., Deits, R., Fallon, M., Valenzuela, A., Dai, H., Permenter, F., \& Tedrake, R. et al. (2016). Optimisation-based locomotion planning, estimation and control design for the atlas humanoid robot. Autonomous Robots, 40(3), 429-455. doi:10.1007/s 10514-015-9479-3

Lauckner, K. F., \& Lintner, M. D. (n.d.). The ROBOT computer: Programs and algorithms. The Computer Continuum. Retrieved from Retrieved from http://guyhaas. com/bfoit/itp/ComputerContinuum/RobotComputer.html 
Raj, M., \& Seamans, R. (2019). Primer on artificial intelligence and robots. Journal of Org. Design, 8(11).

Retto, J. (2017). Sophia, first citizen robot of the world. Retrieved from https://www. researchgate.net/publication/321319964_SOPHIA_FIRST_CITIZEN_ROBOT_OF_ THE_WORLD/citations

Rivlin, O. (2019). Reinforcement learning for real-world robotics. Towards data science. Retrieved from https://towardsdatascience.com/reinforcement-learning-forreal-world-robotics-148c81dbdcff

Rouse, M., Burns, E., \& Laskowski, N. (2018). What is AI (artificial intelligence)? WhatIs.com. Retrieved from https://searchenterpriseai.techtarget.com/definition/AIArtificial-Intelligence

Scassellati, B. (2002). Theory of mind for a humanoid robot. Autonomous Robots, 12(13), 14-24. doi:10.1023/A:1013298507114

Schuman, E. (2015). Are we safe from self-aware robots? Computer World. Retrieved from https://www.computerworld.com/article/2970737/are-we-safe-from-self-awarerobots.html

Sundaresan, Nandhini. (2016). A study on artificial intelligence: The blue brain. International Journal of Innovative Research in Computer and Communication Engineering, 4.

Sunderhauf, N., Brock, O., Scheirer, W., Hadsell, R., Fox, D., Leitner, J., \& Corke, P. et al. (2018). The limits and potentials of deep learning for robotics. The International Journal of Robotics Research, 37(4-5), 405-420. doi:10.1177/0278364918770733 
Naaima Suroor is a final year Bachelor of Technology (CSE) student at Jamia Hamdard with a keen interest in research. She will complete her undergraduate degree in the year 2020.

Imran Hussain has completed a Ph.D. in Computer Science from Jamia Hamdard, New Delhi. He works as an Asst. Professor in SEST, Jamia Hamdard. His research interests include Cloud Computing, Cyber Security, Big data analysis, and CPS.

Aqeel Khalique has completed M.Tech. in CSE from IIT Roorkee. Aqeel works as Asst. Professor in SEST, Jamia Hamdard having around 10 years of experience in academics, industrial research etc. Aqeel is actively involved in research activities and have several publications in the area of Information Security, Pervasive Computing, Cryptology, CPS and Scalable Security. Aqeel is also Associate Editor with renowned journal.

Tabrej Ahamad Khan has completed M. Tech in Information Security from NIT Jalandhar. Tabrej works as Asst. Professor in SEST, Jamia Hamdard having around 5 years of experience in academics and research. Tabrej has a profound interest in the research area such as data analysis, machine learning and social network analysis. 